\title{
First Investigations on Influence of Resolution Cell Size for Monitoring Bare Agricultural Soil by using Ground Based SAR (PoSAR) System Measurements
}

\author{
Sophie Allain*, Stéphane Méric*†, Hongquan Wang* ${ }^{* \dagger}$ and Eric Pottier* \\ ${ }^{*}$ Remote Sensing team, Institute of Electronics and Telecommunications of Rennes (IETR) \\ ${ }^{\dagger}$ INSA of Rennes, European University of Brittany \\ Campus Beaulieu, bldg 11D, 263 av. Général Leclerc, CS 74205, 35042 Rennes Cedex \\ Email: stephane.meric@insa-rennes.fr
}

\begin{abstract}
The objective of this study is to evaluate the influence of resolution cell size of a SAR image in order to monitor bare agricultural soil. In order to achieve this objective, we propose to use Ground Based (GB) SAR experiments, in which the specific levels of surface roughness and soil moisture are set deliberately. For these first investigations, we propose to study the backscattering coefficients $\sigma_{H H}$ for different surface roughness values regarding multi-resolution. These first results we obtained with our GB-SAR system (called PoSAR for Pocket SAR) are in agreement with previous experiments on indoor SAR measurements (JRC dataset).
\end{abstract}

\section{INTRODUCTION}

Polarimetric SAR provides an opportunity to monitor the spatial and temporal distributions of the soil parameters in both regional and continental scale. Especially, polarimetric SAR response from land cover can be significantly influenced by the following two parameter categories: (1) Target parameters, such as roughness, soil moisture, soil texture and vegetation status; and (2) Sensor parameters, such as frequency, polarization and incidence angle. Thus, theoretical or empirical models are normally used to convert the measured backscattering coefficients into soil moisture and surface roughness. Moreover, representative algorithms are established which relate the backscattering coefficients and co/cross polarization ratio directly with surface roughness and soil moisture using semiempirical coefficients. Consequently, on the one hand, retrieval of surface parameters from multi polarimetric SAR measurement has been extensively investigated in the past decades [1]. On the other hand, the multi-angular SAR images have also potentials to improve the estimation of soil parameters [1]. In that way, multi-angular and multi-polarization configuration are compared in [2], indicating the sensitivity of multi-angular configuration to surface roughness is 10 times than multipolarization, which encourages the continuous exploration of multi-angular models. For instance, it was proposed in [1] an original surface roughness parameter to include horizontal and vertical roughness statistical information, and found that backscattering coefficient difference between two SAR acquisitions depends more on surface roughness than soil moisture, in accordance with the results of [2].
Nowadays, operational spaceborne and airborne SAR provide major observational images to monitor soil status. Nevertheless, during the time interval of multi-angular image acquisitions, the surface status (soil moisture or roughness) may changes. Moreover, it is quite difficult to accomplish all the ground truth measurements over large areas at the same time. These are obvious constraints with currently orbiting radar systems which can not acquire multi-angular images at the same time. To overcome this drawback, the Ground Based (GB) SAR system provides an opportunity to gap the spaceborne and airborne SAR system limitations. It is capable of acquiring multi-incidence angle images in quasi-invariant soil and roughness conditions.

In this study, we aim to analyze the influence of resolution cell size on the sensitivity of polarimetric parameters to bare soil. The GB-SAR experiments we achieved are analyzed and firstly compared with the behaviors of indoor polarimetric SAR measurements. Section II describes the GB-SAR PoSAR experiments and the soil conditions we set up for the measurements. In section III, the indoor polarimetric SAR measurements (JRC dataset) we used for the comparison are introduced. Moreover, the influence of SAR image resolution is analysed as well as the soil parameter regarding several roughness conditions. Finally, main conclusions are presented in section IV.

\section{EXPERIMENT DESCRIPTIONS}

\section{A. GB-SAR system measurement}

A very high resolution GB-SAR PoSAR system is developed in the IETR [3]. This system is comprised of vector network analyzer, accurate positioning system, antennas, and 3-m rail which undertakes the system. The signal is generated by vector network analyzer in stepped-frequency mode, and then transmitted during system movement which is controlled by the accurate positioning system. The GB-SAR system obtain each line of the image in a stationary state, thus the Doppler centroid is exactly zero. In addition, the value of Doppler rate can be derived by the geometry configuration between the system and target. These accurate estimations of Doppler parameters is essential for signal synthesis. Two GB-SAR 




(a)



(b)
Fig. 1: (a) Surface roughness $S R F$ measurements; (b) Synchronous samplings using laser and chain.

PoSAR campaigns were carried out over the experimental site of Monterfil (N $48^{\circ} 4^{\prime}, \mathrm{E} 1^{\circ} 58^{\prime}$ ) on 17 July 2013 and on 16 October 2013.

During each GB-SAR PoSAR operation, in situ measurements of soil moisture and surface roughness are implemented. In the one hand, surface roughness was measured by a simple and fast chain approach proposed by [4]. It is based on the justification that when a chain of given length L1 $(146.5 \mathrm{~cm}$ with $2.2 \mathrm{~cm}$ linkage length in our case) is placed straightly along a surface, the covering horizontal distance L2 decreases as surface roughness increases. Therefore, the Saleh Roughness Factor (SRF) is defined as: $S R F=100(1-L 2 / L 1)$. This $S R F$ roughness descriptor is used in our study. The $S R F$ was measured 10 times uniformly in two perpendicular directions so as to represent the entire field Fig. 1(a). Moreover, considering the surface roughness is frequently characterized as random roughness factor ( $s$ :root mean square height of surface profile) measured using laser/pin roughness meter, a transformation relationship $s(\mathrm{~cm})=a S R F^{b}$ is proposed in [5]. The coefficients $a$ and $b$ are in function of rainfall amount [5], and also affected a lot by the linkage length as reported [6]. In our study, the coefficients for roughness scale transformation are derived as $a=0.5072, b=0.7867$ based on the regression between our measurements using laser and synchronous sampling data using chain approach Fig. 1(b). For this roughness measurement validation, four roughness conditions $(k s=1.9,2.8,3.0,3.9$, where $k$ wave number and $s$ standard deviation of surface profile) are set by the tillage operation Fig. 2(a). On the other hand, soil moisture at depth $3.8 \mathrm{~cm}$ was measured using a calibrated Time Domain Reflectometry (TDR) with samples distributed homogeneously over the test site so as to obtain a representative mean soil moisture value. Different soil moisture status are set by using the Deltalab Microprocessor Controlled Spray System, EID 330 that simulates rain events in still air. Rainfall is simulated by a constant speed oscillating nozzle (Deltalab, Tec Jet SS 6560) at a height of $3.8 \mathrm{~m}$ as shown in Fig. 2(b).

In the multi-angular SAR measurement campaign on July, 2013, the GB-SAR system, as shown in Fig. 3(a) is positioned on a tower with a height of around $12 \mathrm{~m}$. The four antennas from left to right shown in Fig. 3(b) correspond V, H, V, H polarization respectively. The angle of Look Of Sight (LOS) is



(a)



(b)
Fig. 2: Different (a) surface roughness; (b) soil moisture setting up in GB-SAR experiments.



(a)

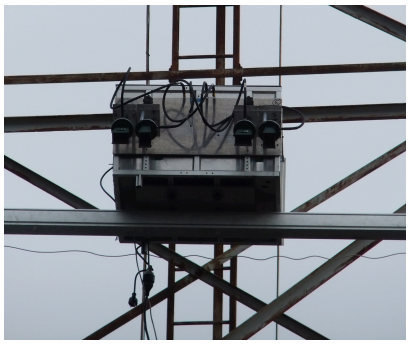

(b)
Fig. 3: (a) PoSAR system on a tower, (b) On site picture

$45^{\circ}$ and the beam width in range direction is $54^{\circ}$ (see Fig. 4). Moreover, a bare soil is selected to carry out this experiment for characterizing different roughness status in a same SAR image (see Fig. 5). The soil moisture can be considered as isotropic over this bare surface. Our measurement is carried out at frequency between $4.9 \mathrm{GHz}$ and $5.9 \mathrm{GHz}$, with 801 frequency samples. On 16 October 2013, the system is mounted on a scaffold with height $6.3 \mathrm{~m}$ as shown in Fig. 6(a). The four antennas from left to right shown in Fig. 6(b) correspond $\mathrm{H}, \mathrm{V}, \mathrm{H}, \mathrm{V}$ polarization respectively. The operating central frequency is set to $5.4 \mathrm{GHz}$ with a $500 \mathrm{MHz}$ bandwidth. The angle of Look Of Sight (LOS) is $38^{\circ}$ and the beam width in range direction is $54^{\circ}$ (see Fig. 7).

\section{SAR DATA ANALYSIS}

At this time, we only present the first results we obtained about the influence of resolution cell size by processing GB-



Fig. 4: Geometric slant configuration in July 


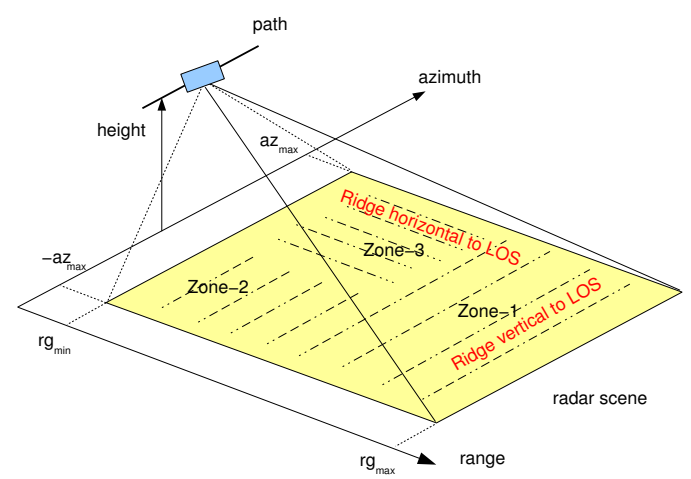

Fig. 5: Soil roughness status



(a)



(b)
Fig. 6: PoSAR system deployment on scaffold.

SAR PoSAR data and regarding the soil properties (roughness under one moisture status of around 20\%).

\section{A. GB-SAR PoSAR data processing}

The received signal is synthesized using back projection algorithm [7] and the basic radar scene which is exhibited in the Fig. 8 is used for polarimetric and radiometric calibrations. Firstly, an antenna correction is applied to compensate the antenna pattern. Then the polarimetric calibration is implemented on the synthesized images using approach [8] to remove the cross talk and channel imbalance. Finally, the radiometric calibration is accomplished by calculating the theoretical radar cross section of trihedral corner reflector [9] and comparing with the integral energy of the trihedral within the image.



Fig. 7: Geometric slant configuration in October

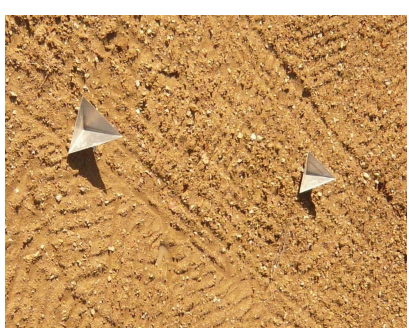

Fig. 8: The trihedral corner reflectors within scene

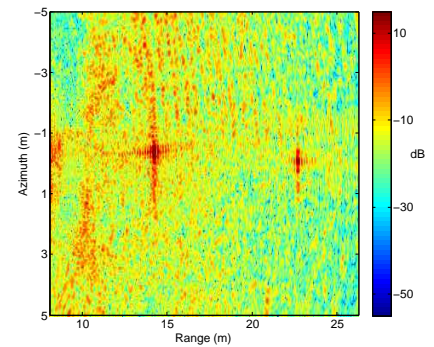

(a)



(b)
Fig. 9: Sar images of the radar scene for (a) July; (b) October.

As shown in Fig. 9, the trihedral corner reflector deployed within the scene is well synthesized with azimuth and range resolution around $15 \mathrm{~cm}$ in July and around $30 \mathrm{~cm}$ in October.

\section{B. JRC dataset}

In order to compare and validate GB-SAR measurements, multi-resolution indoor SAR measurements performed at the European Microwave Signature Laboratory (EMSL) are analyzed. SAR data were acquired for different cell resolution at $14 \mathrm{GHz}$ for an incidence angle of $40^{\circ}$. The target is a smooth isotropic Gaussian surface with the following roughness parameters values: correlation length $L_{c}$ of $6 \mathrm{~cm}$ and rms heights of $0.4 \mathrm{~cm}$ and $2.5 \mathrm{~cm}$ for smooth and rough artificial surface respectively. The resolution values $R$ are equal to $4.2 \mathrm{~cm}$, $4.8 \mathrm{~cm}, 5.4 \mathrm{~cm}, 6 \mathrm{~cm}, 6.6 \mathrm{~cm}, 24 \mathrm{~cm}$. The backscattering power in HH polarization (see Fig. 10) is observed for different resolution sizes. As described in [10], [11], the power decreases with resolution with the same behavior for the two surfaces. Moreover, the observation of two SAR images with spatial

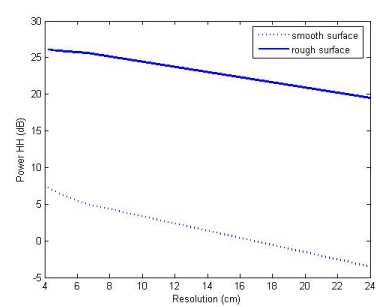

Fig. 10: Backscattering power in JRC datasets. 


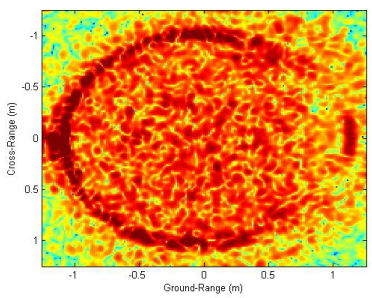

(a)



(b)
Fig. 11: SAR images with JRC datasets



Fig. 12: The backscattering power against resolution cells in our GB-SAR experiments

resolution respectively smaller (see Fig. 11(a)) and larger (see Fig. 11(b)) than $L_{c}$ shows the SAR responses are different. On the left image, the scattering information is distributed on the large scale surface and heterogeneous. As the resolution cell increases, the image become more homogeneous.

\section{Comparisons with our GB-SAR measurements}

The GB-SAR images obtained during the October campaigns are focused with different resolutions by varying the frequency bandwidth and azimuth aperture extent [12]. Four different surface roughness conditions are set: rms height equal to $1.7 \mathrm{~cm}, 2.5 \mathrm{~cm}, 2.7 \mathrm{~cm}$ and $3.5 \mathrm{~cm}$ respectively. In accordance with the behaviors of JRC dataset, the $\mathrm{HH}$ polarization signature in our GB-SAR measurements decreases with resolution (see Fig. 12). However, the behaviors of co polarization signature $\mathrm{HH}$ is different from the JRC dataset and especially concerning the roughness sensitivity. Indeed, the two surface roughness conditions in our GB-SAR experiments are not so sensitive as in JRC dataset. Furthermore, two SAR images in $\mathrm{HH}$ polarization with resolution $30 \mathrm{~cm}$ and $120 \mathrm{~cm}$ are shown in Fig. 13, indicating the energy distributions are more homogeneous at a larger resolution cell. The SAR responses vary due to the resolution cell differences.

\section{CONCLusion}

In this study, multi-resolution polarimetric SAR images acquired by GB-SAR and in anechoid chamber are analyzed to characterize the influence of resolution cell size on radar response over bare surfaces. The main objective of this first study is to validate our new GB-SAR dataset regarding the JRC dataset. Consequently, we can say that both processing



(a)



(b)
Fig. 13: Backscattering power in our GB-SAR experiments

we apply to our radar measurements make possible to deeply study our SAR images from bare surface we can monitor. Also, this first study shows that the reflected power decreases with the resolution. For the further version of this proposal, we plan to study the roughness surface and periodic surface effects on the SAR image with respect the range resolution. Moreover, SAR polarimetric descriptors will be studied in order to retrieve surface parameters in case of agricultural bare soils.

\section{ACKNOWLEDGMENT}

The authors would like to really thank Cécile Leconte and Frédéric Boutet for their help in ground truth measurements.

\section{REFERENCES}

[1] M.Zribi and M.Dechambre, "A new empirical model to retrieve soil moisture and roughness from c-band radar data," Remote Sensing of Environment, vol. 84, pp. 42-52, 2002.

[2] B.Molineaux, A. Chanzy, and M. Zribi, "Using multi-angular radar data to discriminate the influence of rough surface scattering on soil moisture inversions over bare soils," in IGARSS, 1999.

[3] L. Ferro-Famil, C. Leconte, F. Boutet, X. Phan, M. Gay, and Y. Durand, "PoSAR: A VHR tomographic GB-SAR system application to snow cover 3-D imaging at X and $\mathrm{Ku}$ bands," in 9th European Radar Conference (EuRAD), 2012, pp. 130-133.

[4] S. Saleh, "Soil roughness measurements:chain method," J. Soil Water Conserv., vol. 48, pp. 527-529, 1993.

[5] J. E. Gilley and E. R. Kottwitz, "Random roughness assessment by the pin and chain method," Appl Eng Agric, vol. 12, pp. 39-43, 1995.

[6] S. D. Merrill, C. Huang, T. M. Zobeck, and D. L. Tanaka, "Use of chain set for scale-sensitive and erosion-relevant measurements of soil surface roughness," in 10 th International soil conservation organization meeting, 1999.

[7] I. Cumming and F. Wong, Digital processing of synthetic aperture radar data. Artech house, 2005.

[8] T. L. Ainsworth, L. Ferro-Famil, and J. S. Lee, "Orientation angle preserving a posteriori polarimetcalibration SAR calibration," IEEE Trans. Geosci. Remote Sensing, vol. 44, pp. 994-1003, 2006.

[9] J. Van Zyl, "Calibration of polarimetric radar images using only image parameters and trihedral corner reflector responses," IEEE Trans. Geosci. Remote Sensing, vol. 28, no. 3, pp. 337-348, 1990.

[10] G. Nesti, J. Fortuny, and A. Sieber, "Comparison of backscattered signal statistics as derived from indoor scatterometric and sar experiments," Geoscience and Remote Sensing, IEEE Transactions on, vol. 34, no. 5, pp. 1074-1083, 1996.

[11] S. Allain, L. Ferro-Famil, E. Pottier, and J. Fortuny, "Influence of resolution cell size for surface parameters retrieval from polarimetric sar data," in IGARSS, 2003.

[12] H. Wang, S. Meric, S. Allain, and E. Pottier, "Monitoring Bare Agricultural Soil: Comparison between Ground Based SAR PoSAR System Measurements and Multi-angular RADARSAT-2 Datasets," in EUSAR, 2014. 